OSU-TA-2/95

UMN-TH-1325/95

hep-ph/yymmddd

February 1995

\title{
A New Look At Neutrino Limits From Big Bang Nucleosynthesis
}

\author{
Keith A. Olive \\ School of Physics and Astronomy, University of Minnesota \\ Minneapolis, MN 55455, USA \\ and \\ Gary Steigman \\ Department of Physics, The Ohio State University \\ Columbus, $\mathrm{OH} 43210$, USA
}

\begin{abstract}
We take a fresh look at the limits on the number of neutrino flavors derived from big bang nucleosynthesis. In particular, recent measurements of the ${ }^{4} \mathrm{He}$ abundance enable one to estimate the primordial ${ }^{4} \mathrm{He}$ mass fraction at $Y_{p}=0.232 \pm .003($ stat $) \pm .005$ (syst). For a baryon to photon ratio, $\eta$, consistent with the other light elements, this leads to a best fit for the number of neutrino flavors $N_{\nu}<3$ (the precise number depends on $\eta$ ) indicating a very strong upper limit to $N_{\nu}$. Here, we derive new upper limits on $N_{\nu}$, paying special attention to the fact that the best estimate may lie in an unphysical region $\left(N_{\nu}<3\right.$ if all three neutrino flavors are light or massless; the lower bound to $N_{\nu}$ may even be as low as 2 , if the small window for a $\nu_{\tau}$ mass is exploited). Our resulting upper limits therefore depend on whether $N_{\nu} \geq 2$ or 3 is assumed. We also explore the sensitivity of our results to the adopted value of $\eta$ and the assumed systematic errors in $Y_{p}$.
\end{abstract}


Among the strongest cosmological constraints on particle physics models are those derived from the consistency of standard big bang nucleosynthesis (SBBN) and, of particular importance are the constraints determined from the consistency of the predicted and observed primordial ${ }^{4} \mathrm{He}$ mass fraction, $Y_{p}$. Most notable among these constraints is the limit on the number of neutrino flavors [1] which can be translated into a host of other limits on particle physics properties. In the SBBN [2], the abundances are primarily sensitive to a single parameter, the baryon-to-photon ratio, $\eta$. Consistency between the predictions of SBBN and the observational determinations of the light element abundances restricts $\eta$ to a narrow range between $2.8 \times 10^{-10}$ and $4 \times 10^{-10}$ [2]. In this range the calculated ${ }^{4} \mathrm{He}$ mass fraction lies in the range $Y_{p}=0.240-0.245$ [3], which is high when compared with the observationally inferred best primordial value [4],

$$
Y_{p}=0.232 \pm 0.003 \pm 0.005
$$

where the errors are $1 \sigma$ statistical and systematic errors respectively. Indeed, consistency within these small errors allows for very little room for any enhancement in primordial ${ }^{4} \mathrm{He}$. This is the reason that SBBN leads to very tight limits on the number of neutrino flavors [1, 田, 5].

Indeed, based on our estimate for the primordial mass fraction of ${ }^{4} \mathrm{He}$, we deduced a best fit for the number of neutrino flavors [4],

$$
N_{\nu}=2.17 \pm 0.27 \pm 0.42
$$

In [4] we used a $2 \sigma_{\text {stat }}+\sigma_{\text {sys }}$ upper limit for $Y^{\max }$ in order to test the consistency of the SBBN. That procedure yields $N_{\nu}<3.13$, a value similar to, but somewhat weaker than the $95 \%$ CL limit $N_{\nu}<3.04$ from a Monte Carlo analysis [5]. However, if there are indeed three massless neutrinos, then our best fit $\left(N_{\nu}=2.17\right)$ is in fact in the unphysical regime. Limits derived from a probability distribution centered in an unphysical region of parameter space are known to give overly restrictive bounds. 1 In this letter the limits on $N_{\nu}$ are rederived by renormalizing the probability distribution to the physical portion of parameter space. We

\footnotetext{
${ }^{1}$ We thank Paul Langacker for bringing this issue to our attention.
} 
consider both $N_{\nu} \geq 3$ and $N_{\nu} \geq 2$ as criteria for the physical region. The limits on $N_{\nu}$ are of course weakened, even in the latter case $\left(N_{\nu}>2\right)$ where though the mean value is physical, much of the probability distribution still lies in an unphysical region $\left(N_{\nu}<2\right)$.

The best approach to a BBN bound on $N_{\nu}$ is to fit simultaneously both $\eta$ and $N_{\nu}$ to the inferred primordial abundances of all the light elements [6]. Here, instead, we simply explore these new limits to $N_{\nu}$ as a function of $\eta$, as well as of the assumed systematic uncertainty. However, treatment of the systematic uncertainty can not always be done rigorously. We therefore study the implications of two different assumptions. We will assume either that they are Gaussian, in which case they will add in quadrature with the statistical errors or, that they are described by a top-hat distribution which we convolve with the Gaussian statistical errors (this is one of the cases being considered in [3]). We also discuss the consequences of having several sources of systematic errors on our estimates of $N_{\nu}$ and $Y^{\max }$.

As a prelude to our statistical analysis, it is useful to discuss first the relationship between $N_{\nu}$ and the ${ }^{4} \mathrm{He}$ abundance [1]. The ${ }^{4} \mathrm{He}$ abundance is primarily determined by the neutronto-proton ratio just prior to nucleosynthesis or, more accurately, prior to the freeze-out of the weak interaction rates at a temperature $T_{f} \sim 1 \mathrm{MeV}$. The final ${ }^{4} \mathrm{He}$ abundance is in fact quite sensitive to the freeze-out temperature which is determined by the competition between the weak interaction rates and the expansion rate of the Universe. As the uncertainty in the neutron mean-life is now very small, $\tau_{n}=887 \pm 2 \mathrm{~s}$ [7], the helium abundance depends primarily on $\eta$ which determines the onset of nucleosynthesis and $N_{\nu}$, which can be used to characterize the expansion rate of the Universe at $T \gtrsim 1 \mathrm{MeV}$, as measured by the (time dependent) Hubble parameter $\mathrm{H}$,

$$
H=\left(\frac{8 \pi^{2} G_{N}\left(N_{*}+\frac{7}{4} N_{\nu}\right)}{90}\right)^{1 / 2} T^{2}
$$

where $N_{*}=5.5$ provides the contribution from electrons and photons. It is appropriate to emphasize that $N_{\nu}$ measures the contribution to the energy density at the epoch of nucleosynthesis of neutrinos (massive or massless) and any additional particles beyond those in the standard model. In the standard model, $N_{\nu}=3$ (unless the $\tau$ neutrino has a mass in excess of about $0.1 \mathrm{MeV}$ ) [8]. Limits on additional massless neutrino flavors with standard 
model coupling to the $Z$ gauge boson are very tightly constrained by LEP: $N_{\nu}<3.04$ (2 б) [7]. The nucleosynthesis bounds however are most sensitive to any relativistic particle species present at that time. For example, an additional massless scalar degree of freedom such as the majoron, would contribute $4 / 7$ to $N_{\nu}$.

The neutron-proton "freeze-out" is determined by the competition between the expansion rate (3) and the weak interaction rate (proportional to $T^{5}$ ). A larger value for $N_{\nu}$ increases the expansion rate leading to earlier freeze-out at a higher neutron to proton ratio and, hence, yields a higher value for $Y_{p}$. To understand the behavior of the calculated value of $Y_{p}$ as $\eta_{10}, \tau_{n}$ and $N_{\nu}$ are varied, it is useful to fit the SBBN results in the form

$$
Y_{p}=A+B \ln \eta_{10}+C\left(\tau_{n}-887\right)+D\left(N_{\nu}-3\right)
$$

As $\eta_{10}$ increases from 2 to 10 , B decreases from 0.014 to 0.009 . For all $\eta, C=2 \times 10^{-4}$, confirming that the small uncertainty in the neutron lifetime contributes negligibly to the uncertainty in $Y_{p}$. As $N_{\nu}$ varies from 2 to 4 , D decreases from 0.014 to 0.012. From eq. (4), it is clear that to bound $N_{\nu}$ will require that $Y_{p}$ and $\eta_{10}$ and their uncertainties be constrained.

In Yang et al. 9] and Walker et al. [2], we utilized solar system abundances of D and ${ }^{3} \mathrm{He}$ to place an upper bound to the primordial abundance of $\mathrm{D}+{ }^{3} \mathrm{He}$ and we derived a lower bound to $\eta_{10}(>2.8)$. This bound is conservative in the sense that it ignored ${ }^{3} \mathrm{He}$ production in low mass stars. Though models of galactic chemical evolution using primordial abundances near this lower bound on $\eta$ may show sufficient deuterium destruction [10, when ${ }^{3} \mathrm{He}$ production is included these models predict a large excess of ${ }^{3} \mathrm{He}$ [11 unless $\eta$ is large $(\gtrsim 4)$ [12]. Indeed, a recent Monte Carlo analysis [13] found the larger value of $\eta_{10} \sim 6.6 \pm 1.4$ as a best fit even when ${ }^{3} \mathrm{He}$ production was ignored. Thus, the challenge to BBN is clear since, even for $\eta_{10} \geq 2.8$ and $\tau_{n} \geq 885$, we have $Y_{p} \geq 0.240$ for $N_{\nu}=3$. As was stressed in [4], this is only consistent with the observational data if $\sigma(s y s t)=0.005$ is added to the $2 \sigma_{\text {stat }}$ upper bound of 0.238 .

Recently, there have been reports of observations of deuterium in quasar absorption systems 14 with a $\mathrm{D} / \mathrm{H}$ abundance (which may be interpreted to be the primordial one) corresponding to a value of $\eta_{10} \sim 1.5$. Although such a low value for $\eta$ would be consistent with the observed ${ }^{4} \mathrm{He}$ abundance, it would exacerbate the problem due to the overproduction 
of ${ }^{3} \mathrm{He}$ [15, 16]. We note that there is also the possibility that this observation can be interpreted as a $\mathrm{H}$ detection in which the absorber is displaced in velocity by $80 \mathrm{~km} \mathrm{~s}^{-1}$ with respect to the quasar [15]. Such an interpretation is likely, given recent reports [17] that a $\mathrm{D} / \mathrm{H}$ measurement along a different line of sight may indicate a much lower value for $\mathrm{D} / \mathrm{H}$, corresponding to a much higher value for $\eta_{10}(\gtrsim 6)$. If this latter is correct, it would appear to pose a conflict between the BBN predictions and the observational determinations of $Y_{p}$. At this point, however, the data on $\mathrm{D} / \mathrm{H}$ is too limited (and lacks consistency) to permit any firm conclusion. In our subsequent discussion of limits on $N_{\nu}$, we will, where appropriate, give results for several values of $\eta_{10}$.

Before returning to the problem of placing limits on $N_{\nu}$, we first discuss our treatment of errors used in establishing a $2 \sigma$ (or $\sim 95 \%$ CL) upper limit. The chief problem is the treatment of systematic errors. In obtaining $Y^{\max }=0.243$ [ [4], we simply added $\sigma_{\text {sys }}=0.005$ to the $2 \sigma$ statistical upper limit. There are better alternatives. For example, it can be assumed (as is done by the particle data group [7]) that systematic errors are Gaussian distributed and add in quadrature to the statistical errors. In this case, the $2 \sigma$ upper limit to $Y_{p}$ is 0.244 . Or, it may be assumed that the systematic errors are described by a top-hat distribution (ie. constant probability between $\pm \sigma_{\text {sys }}$ and zero otherwise). The convolution of the Gaussian and top-hat distributions is just the difference of error functions given by (unnormalized)

$$
\operatorname{erf}\left(\frac{x+\sigma_{\text {sys }}-\mu}{\sqrt{2} \sigma_{\text {stat }}}\right)-\operatorname{erf}\left(\frac{x-\sigma_{\text {sys }}-\mu}{\sqrt{2} \sigma_{\text {stat }}}\right)
$$

where $\mu=0.232$ is the central value for $Y_{p}$. For $\sigma_{\text {stat }}=0.003$ and $\sigma_{\text {sys }}=0.005$, the distribution given in eq. (5) yields a $95 \%$ CL upper limit of 0.240 which is somewhat more restrictive than the previous two estimates.

It is very difficult to estimate the size of the systematic errors. Indeed, in exploring possible sources of such errors it is important to consider whether they may be correlated or uncorrelated among themselves [3, 60. It has recently been suggested that previous analyses have overlooked potential sources of error and, therefore, that the true systematic uncertainty may be considerably larger than our estimate of 0.005 [18, [19]. Although we do agree that the size of the systematic error remains uncertain, we consider that the recent analyses may 
be overly simplified. To illustrate the problem, consider the contributions to the uncertainty from corrections for ionization and collisional excitation [3, 6]. It is often assumed that there could be some neutral helium in the zone where hydrogen is fully ionized and, therefore, that the ionization correction will only increase the inferred helium abundance [18, 19]. However, this overlooks the fact that very metal-poor stars will tend to be hotter than their higher metallicity counterparts, resulting in a harder ionizing radiation field in the very metal-poor HII regions. In such a situation, the HeII zone may extend beyond the HII zone and an appropriate ionization correction would reduce the inferred He abundance. Even worse, this possible effect would then correlate with the metallicity of the HII region, perhaps reducing $\mathrm{Y}$ at low metallicity and raising it for more metal-rich HII regions. The same considerations apply for the possible correction to account for collisional excitation in helium. This correction (decreasing the inferred helium abundance) will be larger for hotter (metal-poor) regions and smaller for cooler (metal-rich) ones.

Thus, since some of the sources of systematic errors may be correlated (or anticorrelated) and, even their sign uncertain, a naive linear combination of several error estimates [18, 19] must lead to an overestimate of the true uncertainty. To explore the effect of such possible errors on our estimate of $Y^{\max }$, we consider some illustrative examples. For example, let us first assume that the systematic errors are Gaussian distributed. Then we simply add, in quadrature, the systematic error(s) to the statistical one. Thus, as noted above, with our choice of 0.005 for the systematic error and 0.003 for the statistical one, we find for the 95 $\%$ CL upper limit on $Y, Y_{95}=0.244$. For two uncorrelated systematic errors, each of size 0.005 , we would increase this to $Y_{95}=0.247$. If, instead, the systematic error were 0.010 , we would find $Y_{95}=0.253$; note the relatively large difference if the systematic errors are added in quadrature or, linearly. In contrast, if we we treat the systematic errors as uniform ("top-hat"), the probability distribution for $Y$ is given by eq. (5), and for the three cases just considered we find $Y_{95}=0.240$, for a Gaussian statistical error of 0.003 and a top-hat systematic error of $0.005 ; 0.242$, for a Gaussian statistical error of 0.003 and two top-hat systematic errors of 0.005 each (in this case one must convolve a second top hat with the distribution in eq. (5) which is a distribution given by a linear combination of four error functions and four Gaussians); and, 0.244 for a Gaussian statistical error of 0.003 and a 
single top-hat systematic error of 0.010 . These " $2 \sigma$ " upper limits are considerably smaller than those from the double Gaussian approach and certainly much smaller than the upper bound obtained by adding the systematic errors linearly.

We turn now to our computation of the upper limit on $N_{\nu}$. To begin, we take $Y_{p}$ and its uncertainties from eq. (1) and compare to the central predicted value of $Y_{p}$ for each value of $\eta_{10}$ and $\tau_{n}=887 \mathrm{sec}$. to find the best (i.e., central) value for $N_{\nu}$. For example, as $\eta_{10}$ increases from 1 to 10, the central value of $N_{\nu}$ decreases from 3.6 to 1.1 ; the best values of $N_{\nu}$ corresponding to $\eta_{10}=2.8$ and 4.0 , are 2.3 and 1.9 respectively. If all three neutrinos were in fact relativistic at the time of nucleosynthesis, then these latter values (and, indeed, all values for $\eta_{10}>1.5$ ) are unphysical. For the central case of $\eta_{10}=3$, even the $2 \sigma$ statistical upper limit is unphysical. When the systematic uncertainty is included, the upper bound goes above 3.0, but this is most certainly an overly restrictive upper bound based on the data at hand. Here we will use the Bayesian approach described in [0]. Consider first, the double Gaussian treatment of errors (ie., both statistical and systematic errors as Gaussians). In the Gaussian distribution

$$
f(x)=e^{\frac{-\left(x-N_{\nu}\right)^{2}}{2 \sigma^{2}}}
$$

$\sigma^{2}=\sigma_{\text {stat }}^{2}+\sigma_{\text {sys }}^{2}$ and $N_{\nu}$ is the (possibly unphysical) value determined for a given value of $\eta_{10}$. Normally, this distribution would be normalized by integrating over $x$ from $-\infty$ to $\infty$ and setting the result equal to unity. The $95 \%$ CL limits on $x$ would correspond to the limits of integration of the normalized distribution integrated to the value 0.95 . Instead, here we allow the distribution to take non-zero values only over the physical region $\left(N_{\nu} \geq 3\right)$. The distribution is now normalized by integrating from 3 to $\infty$ and the $95 \%$ CL upper limit is the upper limit of integration $x_{95}$ so that the renormalized distribution yields a value 0.95 when integrated from 3 to $x_{95}$,

$$
\frac{\int_{3}^{x_{95}} f(x) d x}{\int_{3}^{\infty} f(x) d x}=0.95
$$

The 95\% CL upper limit on $N_{\nu}$ as a function of $\eta_{10}$ is shown in Figure 1 by the curve labeled $N_{\nu} \geq 3$. For $\eta_{10}>2.8, N_{\nu}<3.61$. This weaker bound would permit a massless scalar.

As it is still possible that $\nu_{\tau}$ is massive and not relativistic at the time of nucleosynthesis, we also show in Figure 1 the result of the same calculation, when the probability distribution 
is allowed to go down to $N_{\nu}=2$ (i.e. the lower limit of integration in eq. (7) is 2 rather than $3)$. In this case, when $\eta_{10}>2.8, N_{\nu}<3.18$, a value closer to but still larger than the value of 3.13 found in the absence of renormalizing the distribution (even though the mean value is now physical, a large portion of the Gaussian is still unphysical). Now, a massless scalar is clearly excluded. It is interesting to note that, due to the renormalization procedure, the limit is actually weaker when three light or massless neutrinos are assumed. This is due to the renormalization procedure.

In Figure 2, we show a similar plot with the systematic errors described by a top-hat distribution. The convolved Gaussian and top-hat is given by eq.(5) where now $\mu$ corresponds to the mean value of $N_{\nu}$ and the errors are the propagated errors in $N_{\nu}$ rather than in $Y_{p}$. The $95 \%$ CL upper limits are found by using the convolved distribution (5) for $f(x)$ in eq. (7). The limits are now somewhat tighter at low $\eta_{10}$. In this case for $\eta_{10}>2.8, N_{\nu}<2.91$ and 3.31 for $N_{\nu} \geq 2$ and 3 respectively, excluding a massless scalar.

Our results clearly depend on the choice of systematic error. In Figures 3 and 4, we show the sensitivity of our results to the value of $\sigma_{\text {sys }}$. We plot the $95 \%$ CL upper limit to $N_{\nu}$ as a function of $\sigma_{\text {sys }}$ for $N_{\nu} \geq 2$ (solid curves) and $N_{\nu} \geq 3$ (dashed curves) for three choices of $\eta_{10}=1.5,2.8$ and 4.0. The curves are arranged such that the limits become more stringent (the upper limit on $N_{\nu}$ is lowered) as $\eta_{10}$ is increased. Figures 3 and 4 correspond to the double Gaussian and Gaussian/top-hat approach described above. Once again the latter provides a more stringent upper bound. As expected, as $\sigma_{\mathrm{sys}}$ is increased the limits on $N_{\nu}$ become significantly weaker.

In conclusion, we have shown that because the best fit values for the number of neutrinolike particle species is, or is close to being unphysical, the "true" upper limits on $N_{\nu}$ are significantly weaker than previous estimates. The upper limit on $N_{\nu}$ is quite dependent on whether one assumes $N_{\nu} \geq 2$ or 3 . Nevertheless, big bang nucleosynthesis can and does supply us with stringent constraint on $N_{\nu}$ and, more generally, on the speed-up of the expansion rate of the Universe. For the perhaps more likely choice of $N_{\nu} \geq 3$, and for $\eta_{10}$ in the range $2.8-4$, the upper limit on $N_{\nu}$ ranges from 3.5 - 3.6 if systematic errors are treated as Gaussian and from $3.2-3.3$ if systematic errors are treated as top-hats. We stress that these limits are only as good as the assumed errors (in particular the systematic errors); 
the limit on $N_{\nu}$ is strongly dependent on $\sigma_{\text {sys }}$. In the case of Gaussian systematic errors, as $\sigma_{\text {sys }}$ approaches 0.01, the bound on $N_{\nu}$ exceeds 4.0 (if $N_{\nu} \geq 3$ ). If the systematic errors are treated as top-hats, then the bound on $N_{\nu}$ is less sensitive and only exceeds 4.0 when $\sigma_{\text {sys }}>.02$. Finally we note that even if the observational errors could eventually be greatly diminished, there remains a residual theoretical error $\leq 0.001$ in $Y$. Because this translates into an error of about 0.08 in $N_{\nu}$, there will always remain a residual uncertainty of order 0.08 in any determination of $N_{\nu}$. Though we have argued here for somewhat weaker bounds on $N_{\nu}$, they nevertheless still provide us with a strong means for limiting particle physics beyond the standard model.

\section{Acknowledgments}

We especially thank Paul Langacker for calling to our attention the key issue we have discussed here and, Naoya Hata, Bob Scherrer, Dave Thomas and Terry Walker for permission to utilize some of the results of our joint work in this manuscript. We also would like to thank T. Falk and M. Roos for helpful conversations. This work was supported in part by DOE grants DE-FG02-94ER40823 and DE-AC02- 76ER01545.

\section{References}

[1] G. Steigman, D.N. Schramm, and J. Gunn, Phys. Lett. B66 (1977) 202.

[2] T.P. Walker, G. Steigman, D.N. Schramm, K.A. Olive and K. Kang, Ap. J. 376 (1991) 51.

[3] D.Thomas, N. Hata, R.G. Scherrer, G. Steigman, G. \& Walker, T.P. 1995, (in preparation).

[4] K.A. Olive and G. Steigman, Ap.J. Supp. 97 (1995) in press.

[5] P. Kernan and L.M. Krauss, Phys. Rev. Lett. 72 (1994) 3309. 
[6] N. Hata, R. J. Scherrer, G. Steigman, D. Thomas, T. P. Walker, S. Bludman and P. Langacker, (1995) in preparation.

[7] Review of Particle Properties, Phys. Rev. D50 (1994) 1173.

[8] E. W. Kolb and R. J. Scherrer, Phys. Rev D25 (1982) 1481.

[9] J. Yang, M.S. Turner, G. Steigman, D.N. Schramm, and K.A. Olive, Ap.J. 281 (1984) 493.

[10] E. Vangioni-Flam, K.A. Olive, and N. Prantzos, Ap.J. 427 (1994) 618; S. Scully and K.A. Olive, Ap.J. (in press) 1995.

[11] K.A. Olive, R.T. Rood, D.N. Schramm, J.W. Truran, and E. Vangioni-Flam, Ap.J. (in press) 1995; M. Tosi, G. Steigman, and D.S.P. Dearborn, in Proceedings of the ESO/EPIC Workshop on the Light Element Abundances, ed. P. Crane (1994).

[12] G. Steigman and M. Tosi, Ap.J. 401 (1992) 15; OSU preprint OSU-TA-12/94 (1994).

[13] N. Hata, R.J. Scherrer, G. Steigman, D. Thomas, and T.P. Walker, astro-ph/9412087, (1994).

[14] R.F. Carswell, M. Rauch, R.J. Weymann, A.J. Cooke, and J.K. Webb, MNRAS 268 (1994) L1; A. Songaila, L.L. Cowie, C. Hogan, and M. Rugers, Nature 368 (1994) 599.

[15] G. Steigman, MNRAS, 269 L53.

[16] E. Vangioni-Flam and M. Cassé, Ap.J. (in press) 1995.

[17] D. Tytler, talk given at the Munich Absorption Line Meeting, (1994).

[18] D. Sasselov and D. Goldwirth, preprint 1994.

[19] C.J. Copi, D.N. Schramm, and M.S. Turner, Science (in press) 1995. 


\section{Figure Captions}

Figure 1: The $95 \%$ CL upper limit on $N_{\nu}$ as a function of $\eta_{10}$ assuming that both statistical and systematic errors are Gaussian distributed. The two curves correspond to the physical condition that $N_{\nu}>2$ or $>3$. In the former case it must be assumed that $\nu_{\tau}$ has a mass near its experimental upper limit.

Figure 2: As in Figure 1, assuming that the systematic errors are described by a top-hat distribution.

Figure 3: The $95 \%$ CL upper limit on $N_{\nu}$ as a function of the systematic uncertainty in $Y_{p}$. The solid (dashed) curves correspond to the condition that $N_{\nu}>2(3)$. Each of these two cases is shown for three choices of $\eta_{10}: 1.5,2.8$, and 4.0. The smaller values of $\eta$ yield weaker upper limits .

Figure 4: As in Figure 3, assuming that the systematic errors are described by a top-hat distribution. 


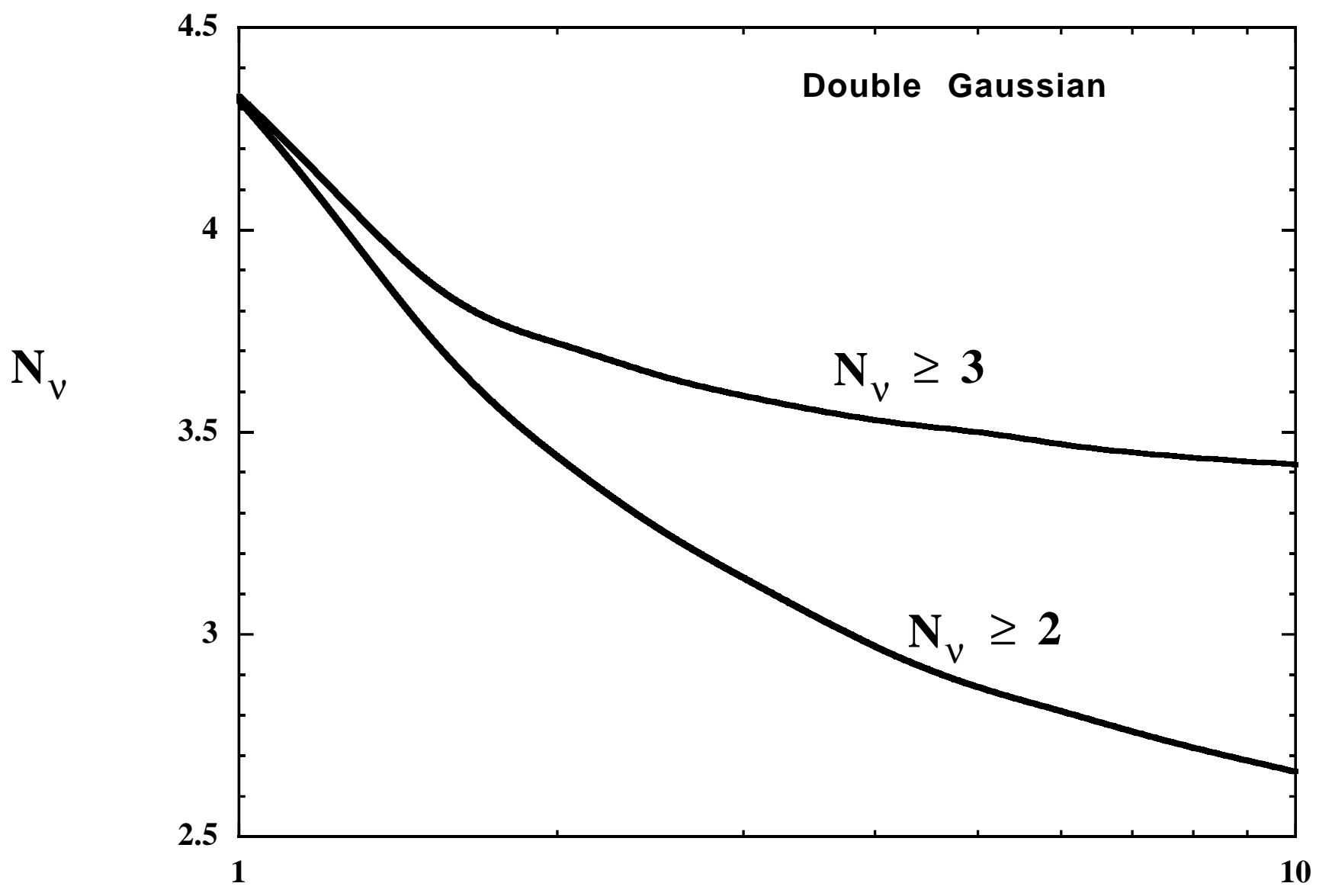

$\eta_{10}$ 


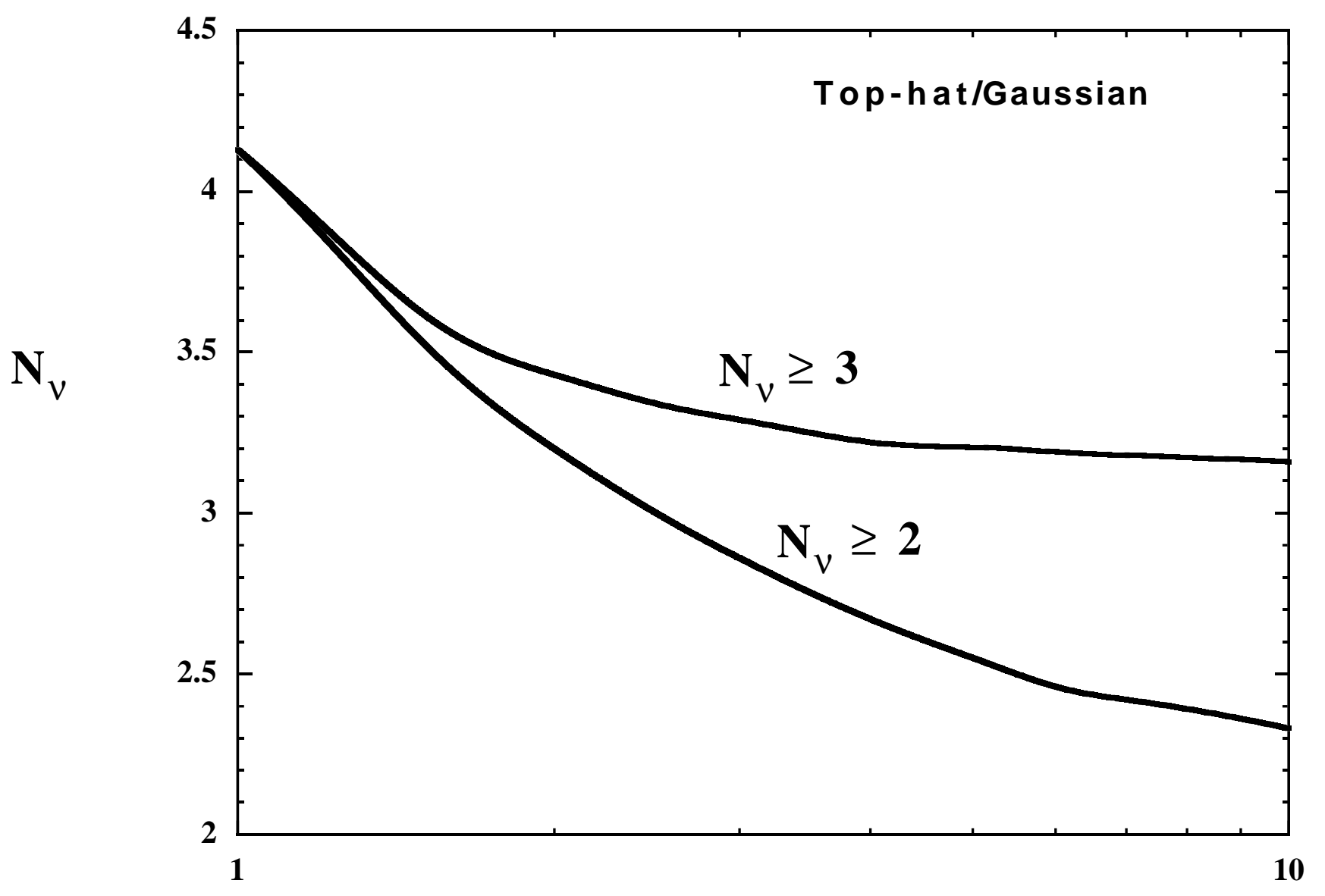

$\eta_{10}$ 


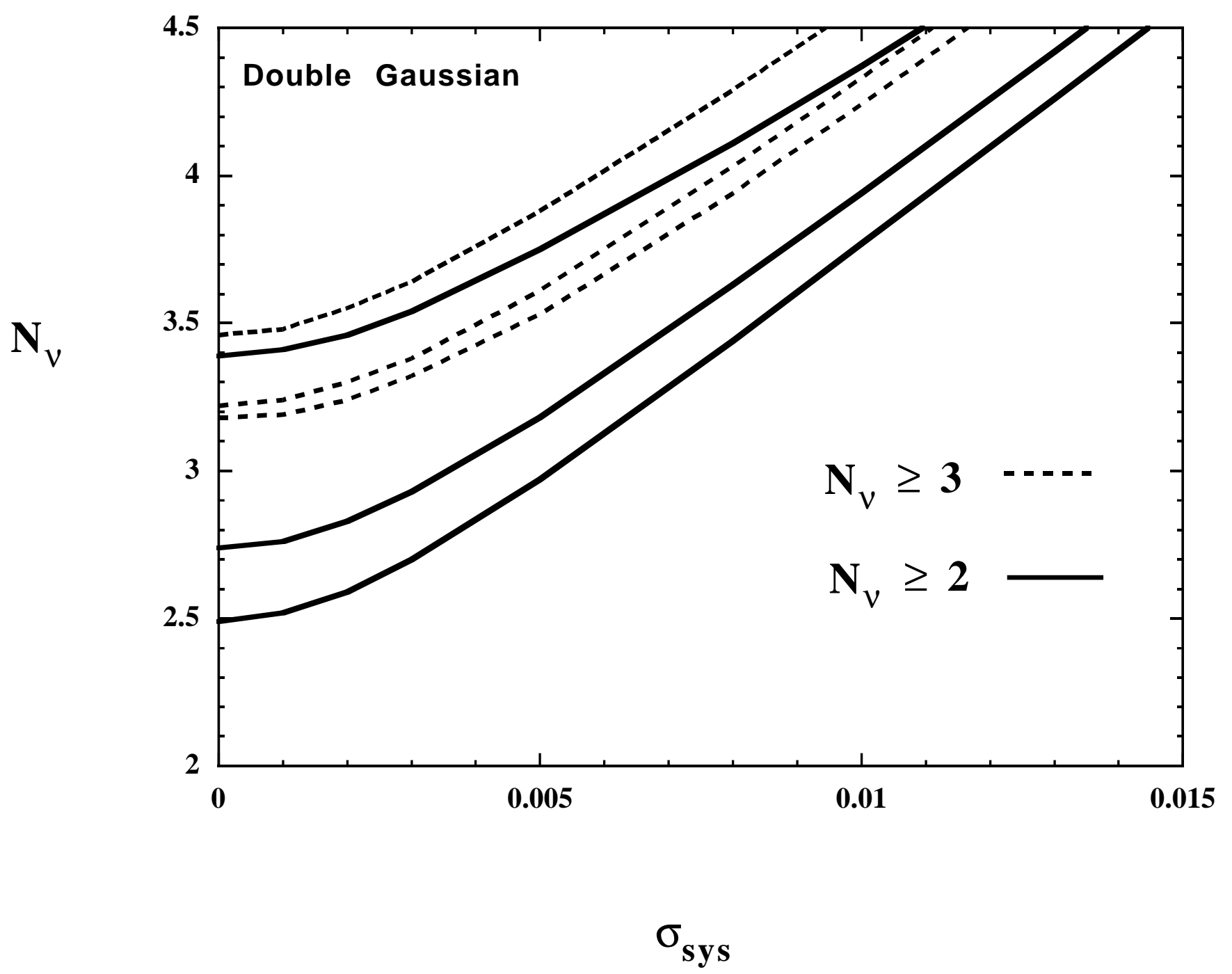




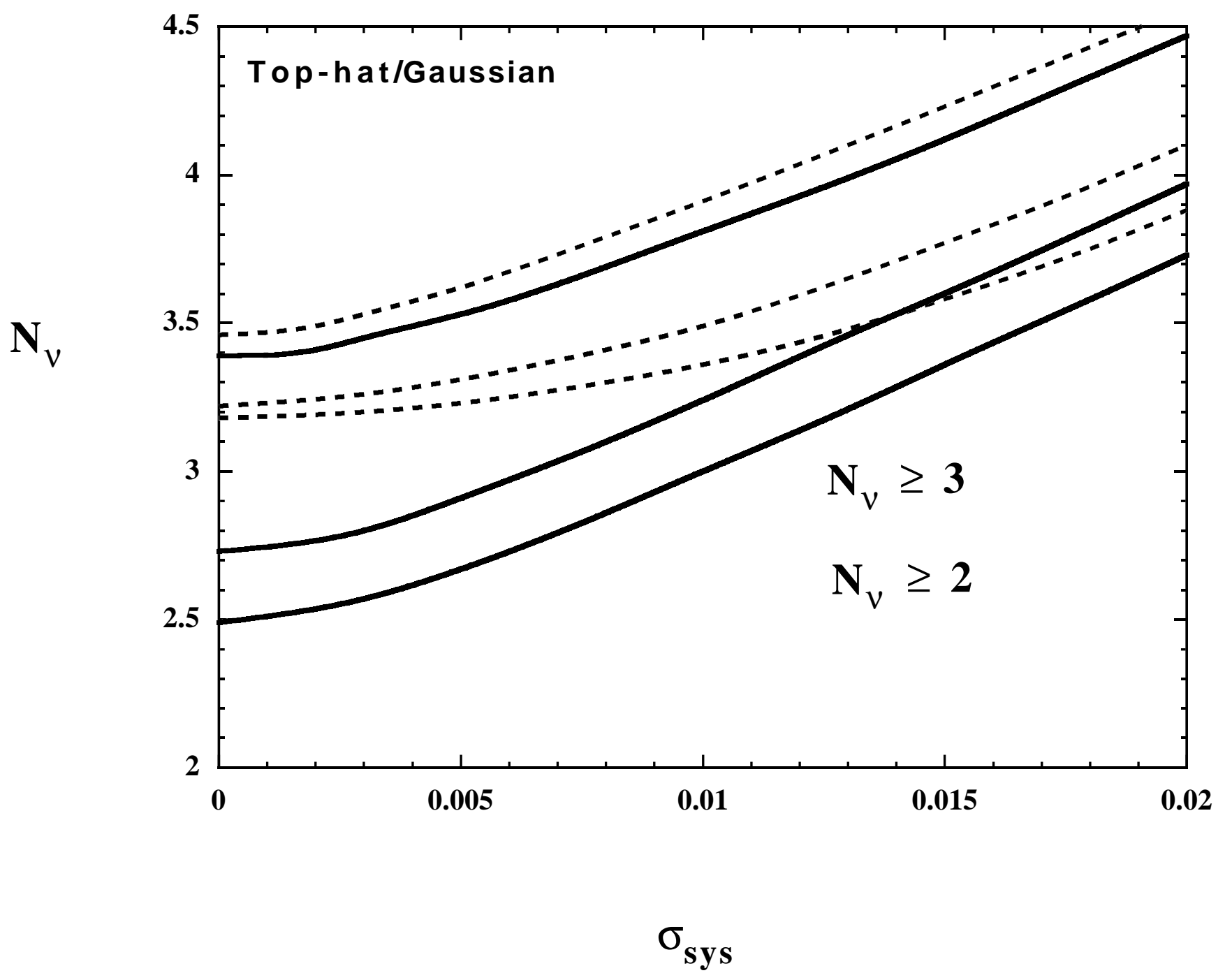

